PROCEEDINGS OF THE

AMERICAN MATHEMATICAL SOCIETY

Volume 103, Number 1, May 1988

\title{
POINT SINGULARITIES AND CONFORMAL METRICS ON RIEMANN SURFACES
}

\author{
ROBERT C. MCOWEN
}

(Communicated by Irwin Kra)

\begin{abstract}
Given a closed hyperbolic Riemann surface and a finite number of points, we prove the existence and uniqueness of hyperbolic conformal metrics with prescribed singularities or degeneracies at the given points.
\end{abstract}

If $M$ is a closed Riemann surface with negative Euler characteristic $\chi(M)$, then it admits a compatible metric $g$ with Gauss curvature $K \equiv-1$. If $p \in M$, then we can ask for a compatible metric $\hat{g}$ on $\hat{M}=M \backslash\{p\}$ with Gauss curvature $\hat{K} \equiv-1$ and some prescribed singularity or degeneracy at $p$,

$$
\hat{g} / g=O\left(r^{2 \alpha}\right) \quad \text { as } r=r(x)=\operatorname{dist}_{g}(x, p) \rightarrow 0 .
$$

Such singularities arise, for example, from maps which are locally $z \rightarrow z^{m}(z \in \mathbf{C}$, $\left.m \in \mathbf{Z}^{+}\right)$: pushing the standard metric forward gives a singularity corresponding to $\alpha=-(m-1) / m$ and pulling back the standard metric gives a degeneracy corresponding to $\alpha=m-1$. Thus we are particularly interested in (1) with $-1<\alpha<\infty$.

More generally, we can consider a finite number of points $p_{1}, \ldots, p_{n} \in M$ and $\alpha_{1}, \ldots, \alpha_{n} \in \mathbf{R}$ and try to find a compatible metric $\hat{g}$ on $\hat{M}=M \backslash\left\{p_{1}, \ldots, p_{n}\right\}$ with

$$
\hat{g} / g=O\left(r_{i}^{2 \alpha_{i}}\right) \quad \text { as } r_{i}=r_{i}(x)=\operatorname{dist}_{g}\left(x, p_{i}\right) \rightarrow 0 .
$$

Our main result is the following.

THEOREM. Let $(M, g)$ be a compact Riemann surface with Gauss curvature $K \equiv$ -1 and $p_{1}, \ldots, p_{n} \in M$. Suppose the numbers $\alpha_{1}, \ldots, \alpha_{n}$ satisfy (i) $-1<\alpha_{i}<\infty$, and (ii) $\chi(M)+\sum_{i} \alpha_{i}<0$. Then $\hat{M}=M \backslash\left\{p_{1}, \ldots, p_{n}\right\}$ admits a unique metric $\hat{g}$ which is pointwise conformal to $g$, has Gauss curvature $\hat{K} \equiv-1$, and satisfies (2). Moreover, $\hat{g}$ has total curvature

$$
\int_{M}(-1) d \hat{A}=2 \pi\left(\chi(M)+\sum_{i} \alpha_{i}\right) .
$$

PROOF. We shall assume for notational convenience that $n=1$ but all steps of the proof generalize immediately. We want to solve

$$
\Delta u-e^{2 u}=-1 \quad \text { on } \hat{M}=M \backslash\{p\}
$$

Received by the editors November 14, 1986 and, in revised form, February 24, 1987. 1980 Mathematics Subject Classification (1985 Revision). Primary 30F10; Secondary 35J60. Key words and phrases. Conformal metrics, Riemann surfaces, point singularities. 
with $u \in C^{\infty}(\hat{M})$ of the form $u=u_{1}+v$ where $u_{1}$ is singular, behaving like $\alpha \ln r$ as $r \rightarrow 0$, and $v$ is bounded; in fact we will have $v \in C^{\beta}(M)$ for some $\beta>0$, indeed $v \in C^{2}(M)$ if $\alpha>0$. Then $\hat{g}=e^{2 u} g$ satisfies (1).

To define $u_{1}$, let $\delta$ be so small that $D=\{x \in M: r(x)<2 \delta\}$ is a disk and let $G(x)$ be the Green's function for $D$ with singularity at $p$, so $G(x)=-(2 \pi)^{-1} \ln (r(x))+$ $O(1)$ as $r(x) \rightarrow 0$. Choose $u_{1} \in C^{\infty}(\hat{M})$ so that $u_{1}(x)=-2 \pi \alpha G(x)$ for $r(x)<\delta$. Notice that

$$
\int_{M} \Delta u_{1} d A=-2 \pi \alpha
$$

We now must find a bounded solution $v$ of

$$
\Delta v=H e^{2 v}-\Delta u_{1}-1 \text { on } M
$$

where $H(x)=\exp \left[2 u_{1}(x)\right]=O\left(r^{2 \alpha}\right)$ as $r \rightarrow 0$. We can reduce this problem even further. Let $u_{0} \in C^{\infty}(M)$ satisfy

$$
\Delta u_{0}=-1-\Delta u_{1}-2 \pi(\chi(M)+\alpha)
$$

(notice the right-hand side has integral zero). If we let $w=v-u_{0}$ and $c=$ $2 \pi(\chi(M)+\alpha)$, then we want to solve

$$
\Delta w=c+h e^{2 w} \quad \text { on } \hat{M}
$$

where $h=H \exp \left[2 u_{0}\right]>0, h=O\left(r^{2 \alpha}\right)$ as $r \rightarrow 0$, and $c<0$ by (ii).

The form of this equation is familiar from [2], and the method of upper and lower solutions employed in [2] to solve it need only be modified slightly to allow the mild singularity of $h$.

By (i) we find $h \in L^{p}(M)$ for $1 \leq p<p_{0}$ where $p_{0}=\infty$ (if $\alpha>0$ ) or $p_{0}=-1 / \alpha$ (if $-1<\alpha<0)$. Thus we may define $\bar{h}=\left(\int h d A\right) /\left(\int d A\right)$ and solve $\Delta \varphi=h-\bar{h}$ with $\varphi \in H_{2}^{p}(M)$ and so $\varphi \in C^{\beta}(M)$ for some $\beta>0$; in particular, $\varphi$ is bounded. Choose $a$ and $b>0$ so that $\bar{h}>-c / a$ and $\exp [2 a \varphi+2 b]>a$. Then $w_{+}=a \varphi+b$ satisfies $\Delta w_{+}<c+h e^{2 w_{+}}$. Now let $k(x)=\max (1, h(x))$. and $\mu>0$ so that $\bar{k}=-c / \mu$. Then we can solve $\Delta \psi=\mu k+c$ with $\psi \in H_{2}^{p}(M)$ for all $p$, and so $\psi \in C^{1+\beta}(M)$ for all $0<\beta<1$; in particular, $\psi$ is bounded. Choose $\lambda$ sufficiently large that $w_{-}=\psi-\lambda$ satisfies $w_{-}<w_{+}$and $\Delta w_{-}>c+h e^{2 w_{-}}$.

REMARK. If $\alpha<0$ we could take $w_{+}=$large positive constant whereas if $\alpha>0$ then we could take $w_{-}=$large negative constant; however this fails if $n>1$ and the $\alpha_{i}$ having varying sign.

We cannot apply Lemma 9.3 of [2] directly since we will not have $w_{+} \in C^{1}(M)$ if $-1<\alpha \leq-1 / 2$. However, $w_{ \pm} \in C^{1}(\hat{M})$ so we can apply the monotone iteration scheme on a sequence of closed sets $M_{j}$ with $\hat{M}=\bigcup M_{j}$ (cf. the proof of Theorem 1 in [1]). We obtain a bounded solution $w$ of $(7)$. Since $h \in C^{\infty}(\hat{M}) \cap L^{p}(M)$ for $1 \leq p<p_{0}$, elliptic regularity shows $w \in C^{\infty}(\hat{M}) \cap C^{\beta}(M)$ for some $\beta>0$ (in fact $w \in C^{2}(M)$ if $\left.\alpha>0\right)$. If we let $S_{\delta}(p)=\{x \in M: r(x)=\delta\}$ and $\nu$ denote the unit normal (towards $p$ ) then

$$
\int_{M} \Delta w d A=\lim _{\delta \rightarrow 0} \int_{S_{\delta}} \frac{\partial w}{\partial \nu} d \sigma=0 .
$$


Thus $u=u_{1}+u_{0}+w$ solves (4); moreover

$$
\int_{M}(-1) d \hat{A}=\int_{M}(-1) e^{2 u} d A=\int_{M}(-1-\Delta u) d A
$$

yields (3) by (5) and (8).

To verify uniqueness, suppose $\hat{g}_{1}$ and $\hat{g}_{2}$ are 2 such metrics. Since they both satisfy (1) this means that there are 2 solutions, $w_{1}$ and $w_{2}$, of (7). If $\alpha>0$ then $w_{1}, w_{2} \in C^{2}(M)$ so $w_{1}=w_{2}$ by the maximum principle. Otherwise, let $w_{0}=w_{1}-w_{2}$ which satisfies $\Delta w_{0}=h\left(e^{2 w_{1}}-e^{2 w_{2}}\right)$ and $w_{0} \in C^{\infty}(\hat{M}) \cap C^{\beta}(M)$. Although $w_{0}$ achieves its maximum and minimum on $M$, it cannot achieve a positive maximum or negative minimum on $\hat{M}$ by the maximum principle. So suppose $w_{0}$ achieves a positive maximum at $p$ : for some small neighborhood $U$ of $p$ we have $w_{0}(x)>0$ for $x \in U$ and $\partial w_{0} / \partial \nu \leq 0$ on $\partial U$ where $\nu$ is the outward normal on $\partial U$. But, arguing as in (8), we find

$$
0<\int_{U} \Delta w_{0} d A=\int_{\partial U} \frac{\partial w_{0}}{\partial \nu} d \sigma \leq 0
$$

a contradiction. Similarly we find that we cannot have a negative minimum at $p$. Thus $w_{1}=w_{2}$ and the metric $\hat{g}$ is unique.

REMARK. Note that the metric $\hat{g}$ is incomplete on $\hat{M}$ by (i). In fact, if $\alpha \leq-1$ then it can be shown (e.g. by an argument similar to the proof of Lemma B in [3]) that (6) admits no bounded solution. Hence condition (i) is necessary, i.e. $g$ admits no complete conformal metric $\hat{g}$ satisfying $(1)$ and $\hat{K} \equiv-1$. On the other hand, by uniformization theory, it is known that $(\hat{M}, g)$ does admit a complete conformal metric $\hat{g}$ with $\hat{K} \equiv-1$. In fact, if we replace $g$ by a conformal metric which is Euclidean near $p$, i.e. $g=d x^{2}+d y^{2}$ and $K \equiv 0$ near $p$, then it is possible to solve $\Delta u-e^{2 u}=K$ on $\hat{M}$ with $u \geq-\ln r-\ln |\ln r|-C$ as $r=\left(x^{2}+y^{2}\right)^{1 / 2} \rightarrow 0$ where $C$ is a constant. Thus (1) is replaced by $\hat{g} / g=O\left(r^{-2}|\ln r|^{-2}\right)$ as $r \rightarrow 0$.

ACKNOWLEDGMENTS. The author wishes to thank K. Uhlenbeck and W. Goldman for bringing this problem to his attention, and UCSD where the work was conducted.

\section{REFERENCES}

1. P. Aviles and R. McOwen, Conformal deformations of complete manifolds with negative curvature, J. Differential Geom. 21 (1985), 269-281.

2. J. Kazdan and F. Warner, Curvature functions for compact 2-manifolds, Ann. of Math. (2) 99 (1974), 14-47.

3. R. McOwen, On the equation $\Delta u+K e^{2 u}=f$ and prescribed negative curvature in $\mathbf{R}^{2}$, J. Math. Anal. Appl. 103 (1984), 365-370.

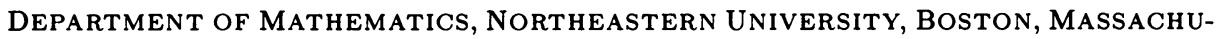
SETTS 02115 